\title{
Prevalência de agenesia do osso sesamóide da articulação metacarpofalangeana, em brasileiros adultos
}

\author{
Renata Rodrigues de Almeida-Pedrin*, Orivaldo Tavano**, Renato Rodrigues de Almeida***,

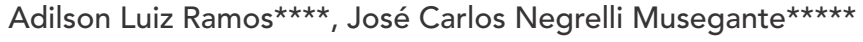

\begin{abstract}
Resumo
Objetivo: diante da importância do sesamóide no emprego de métodos simplificados para a determinação da maturidade esquelética, julgamos necessário um estudo, em brasileiros adultos, para verificar a porcentagem de presença ou ausência deste ossículo. Metodologia: a amostra constitui-se de 500 indivíduos brasileiros, com idade média de 22,5 (18-33) anos, de ambos os gêneros. A presença ou ausência do osso sesamóide foi verificada mediante a interpretação de radiografias periapicais, tomadas da articulação metacarpofalangena dos dois polegares de cada indivíduo da pesquisa. Resultados e Conclusão: os resultados demonstraram que o osso sesamóide da articulação metacarpofalangeana esteve presente em todos os indivíduos (100\%) da amostra, concluindo, desta forma, que não houve agenesia deste osso nesta população avaliada.
\end{abstract}

Palavras-chave: Índice de maturação. Idade esquelética. Osso sesamóide. Radiografia carpal.

\section{INTRODUÇÃO E REVISÃO DE LITERATURA}

O surto de crescimento das dimensões faciais, acompanhando o desenvolvimento corpóreo, constitui um importante aliado do ortodontista para a correção das más oclusões, principalmente da Classe II, divisão 1 , por retrognatismo mandibular.

O uso de aparelhos ortopédicos de avanço mandibular nesta fase de mais rápido crescimento tem sido relatado como mais eficiente (melhor relação tempo-custo), além de contar com melhor perspectiva de estabilidade, dependente da qualidade de intercuspidação dos dentes permanentes $^{8,9}$. Os eventos físicos podem ser parâmetros de localização desta melhor fase de crescimento, mas apesar de apresentarem-se numa seqüência relativamente constante, as idades em que se manifestam variam sensivelmente entre os jovens. Portanto, a idade cronológica não constitui um

Especialista em Radiologia pela FOB-USP. Mestre, Doutora e Pós-Doutorado em Ortodontia pela Faculdade de Odontologia de Bauru-USP. Professora de Especialização em Ortodontia da Faculdade de Odontologia de Lins - UNIMEP e da Uningá-Bauru/CORA.

Mestre e Doutor em Diagnóstico Oral - USP. Professor Titular de Radiologia da FOB/USP. Professor do curso de pós-graduação em Odontologia, subárea Radiologia, da CPO São Leopoldo Mandic.

** Professor associado do departamento de Ortodontia, Odontopediatria e Saúde Coletiva da Faculdade de Odontologia de Bauru-USP. Professor e Coordenador dos cursos de Especialização em Ortodontia da Uningá-Bauru/CORA.

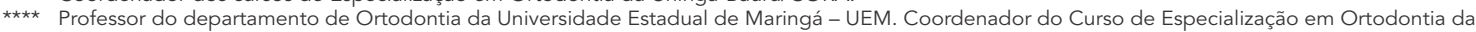
UEM.

UEM. Radiologia Odontológica e Imaginologia da ABENO-SP. 
parâmetro confiável para a determinação do estágio de crescimento. Desta forma, torna-se necessária a observação de outros eventos que possam indicar o grau de maturação biológica ${ }^{3,6}$. A idade dentária ou, neste caso, o início da fase de dentadura permanente seria uma fase bem conveniente para este tipo de tratamento, entretanto, também tem sido demonstrada uma grande variabilidade em comparação à idade biológica de crescimento corpóreo e das dimensões faciais ${ }^{10}$. Entre os métodos para a qualificação da fase do crescimento, a analise do grau de maturação esquelética, mediante a observação direta da radiografia carpal e, mais recentemente explorada, a maturação das vértebras cervicais, têm-se mostrado mais prática e confiável ${ }^{5,13,14}$.

Os métodos que foram descritos para a avaliação da idade biológica utilizando radiografia carpal ${ }^{5,7}$ norteiam-se pela comparação de imagens dos centros de ossificação dos 8 ossos do carpo (escafóide, semilunar, piramidal, pisiforme, trapézio, trapezóide, capitato e hamato), antes da puberdade. Entretanto, com a aproximação da puberdade, as imagens dos ossos carpais confundem-se, dificultando sua visualização. Como alternativa, utiliza-se a comparação das alterações ao nível das falanges, por sua precisão e simplicidade ${ }^{14}$.

Pesquisas recentes avaliaram a maturação esquelética por meio das alterações das vértebras cervicais observadas na telerradiografia em norma lateral, concluindo que a observação das mudanças morfológicas que ocorrem nas vértebras cervicais, visualizadas nas telerradiografias laterais, constitui um método útil e aplicável na avaliação do estágio de maturação de um paciente na prática clínica ${ }^{14}$. Recentemente Bacetti, Franchi e McNamara ${ }^{1}$, baseados nas alterações das vértebras cervicais, apresentaram uma simplificação do método, citando 5 estágios de maturação.

Silva Filho et al. ${ }^{14}$ apresentaram um método simplificado para avaliação apenas da região metacarpofalangeana do $1^{\circ}$ dedo, onde a visualização do ossículo sesamóide e as fases do capeamento epifisário fornece dados suficiente para um bom enquadramento do paciente em relação à curva estatural. O sesamóide adutor ou ulnar, da articulação metacarpofalangeana do polegar surge por volta do início do período da adolescência e guarda uma estreita relação com o início do surto máximo de crescimento estatural e mandibular, além de mostrar uma íntima relação com o início dos caracteres sexuais secundários, com a menarca e com a erupção do segundo molar permanente inferior ${ }^{4,14}$.

Normalmente, o aparecimento do sesamóide precede ou coincide com o surto de crescimento estatural, ocorrendo, em média, de 6 meses a 1 ano antes do pico máximo de crescimento estatural. $\mathrm{O}$ aparecimento do sesamóide, portanto, demonstra que o paciente encontra-se numa época favorável para o tratamento com aparelhos ortopédicos, visando estimular o crescimento mandibular. Embora alguns autores ${ }^{9,11}$ tenham observado variações na época do surgimento desse ossículo nas radiografias, que em alguns casos aparece logo após o pico máximo de crescimento mandibular, o sesamóide constitui um dado de fácil leitura e aplicação clínica, razoavelmente confiável.

Os primeiros a sugerirem a utilização do filme periapical para detectar a presença do sesamóide e o estágio de capeamento epifisário da articulação metacarpofalageana foram Bjork e Helm², que relataram que a presença do sesamóide ocorre em, praticamente, $100 \%$ da população. Após ter sido sistematizado, o método da radiografia periapical foi comparado com o método clássico de Greulich e Pyle ${ }^{7}$. Os resultados mostraram que ambos os métodos permitem um adequado diagnóstico da fase de maturidade esquelética ${ }^{12}$.

A observação de alterações físicas circumpuberais apresentadas pelo paciente (tais como: pelos faciais, alteração da voz, pubarca, telarca e menarca), associada à leitura da radiografia periapical do polegar, fornece dados bastante seguros para o uso clínico e em pesquisas ${ }^{12,14}$.

Com relação à presença do sesamóide, julga-se 
que todos os pacientes, em algum momento, o terão presente na radiografia ${ }^{2}$. Entretanto, Pileski et al. ${ }^{11}$ detectaram uma prevalência de $0,5 \%$ de agenesia deste ossículo, em uma amostra de 199 adultos com mais de 20 anos de idade, numa população canadense (Burlington Orthodontic Research Centre).

Ainda não havia sido estudada uma população brasileira adulta quanto ao percentual de presença ou ausência desse ossículo, que foi a proposta do presente trabalho.

\section{MATERIAL E MÉTODOS}

A amostra constituiu-se de 500 indivíduos brasileiros, com idade média de 22,5 anos (variando entre 18 e 33 anos), de ambos os gêneros (Gráf. 1). Todos os componentes da amostra eram estudantes da Faculdade de Odontologia de Lins e provenientes de diversos estados brasileiros, principalmente das regiões sudeste, sul e centro-oeste. A amostra foi subdividida em dois grupos - leucodermas, com 437 indivíduos e xantodermas, composto por 63 indivíduos (Tab. 1, Gráf. 2) estabelecendo-se um valor numérico por cálculo simples de porcentagem e determinação dos correspondentes intervalos de confiança. A presença ou ausência do sesamóide adutor foi verificada mediante a interpretação de radiografias periapicais, tomadas da articulação metacarpofalangena dos dois polegares de cada indivíduo da amostra.

Utilizou-se um aparelho de raios-X modelo Panpas 3, fabricado pela Funk (Leverkusen, Alemanha), regulado para uma dosagem de radiação ionizante de $50 \mathrm{kVp}$ e $10 \mathrm{~mA}$. As películas radiográficas utilizadas foram da série Ektaspeed EP-21, fabricadas pela AgfaGevaert (Morstel, Bélgica).

Para as tomadas radiográficas foi desenvolvido um equipamento (uma caixa) para padronização e facilidade de produção rápida em série. A caixa constituída de madeira possuía duas aberturas frontais para posicionar as mãos (Fig. 1), um orifício na parte superior para encaixar o cilindro do aparelho de raios-X (Fig. 2) e a parte poste- rior totalmente aberta, para facilitar o acesso do operador (Fig. 3). No assoalho interno da caixa foi adaptada uma placa de isopor, com a finalidade de facilitar o posicionamento (em altura) das mãos e proporcionar conforto ao componente da amostra (Fig. 4). A parte interna frontal e lateral da caixa foi revestida com uma lâmina de chumbo, para assegurar proteção contra radiações ionizantes (Fig. 3). Ressalta-se, ainda, que todos os componentes da amostra utilizaram colete de chumbo durante a tomada radiográfica. A participação dos integrantes foi voluntária, sob consentimento livre e infor-

Tabela 1 - Distribuição dos componentes da amostra.

\begin{tabular}{cccc}
\hline & leucodermas & xantodermas & total \\
\hline feminino & 294 & 35 & 329 \\
masculino & 143 & 28 & 171 \\
subtotal & 437 & 63 & 500 \\
\hline
\end{tabular}

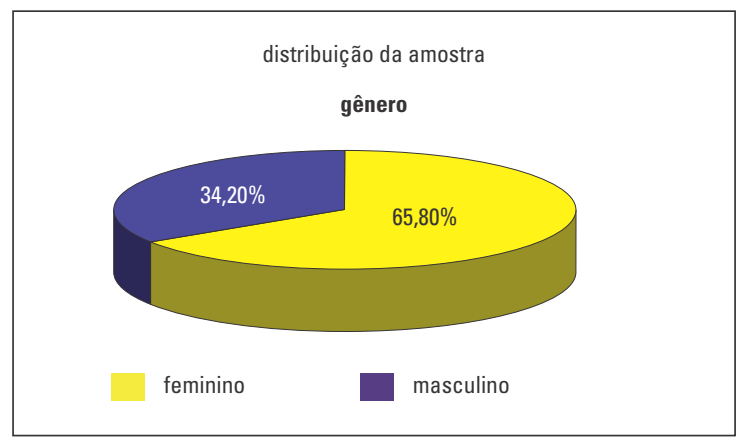

GRÁFICO 1 - Distribuição da amostra segundo gênero.

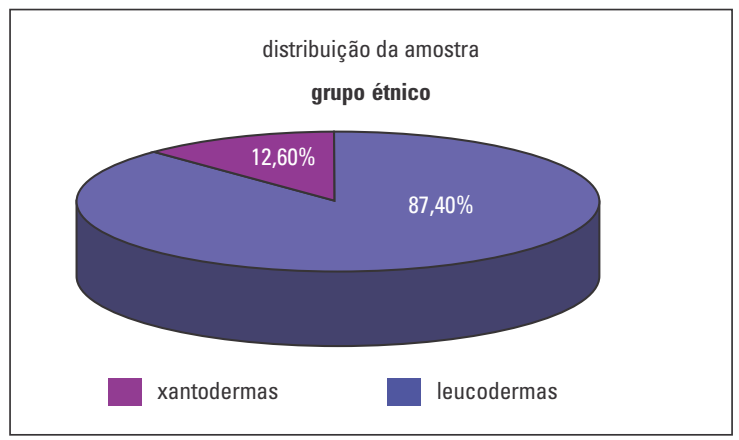

GRÁFICO 2 - Distribuição da amostra segundo grupo étnico. 


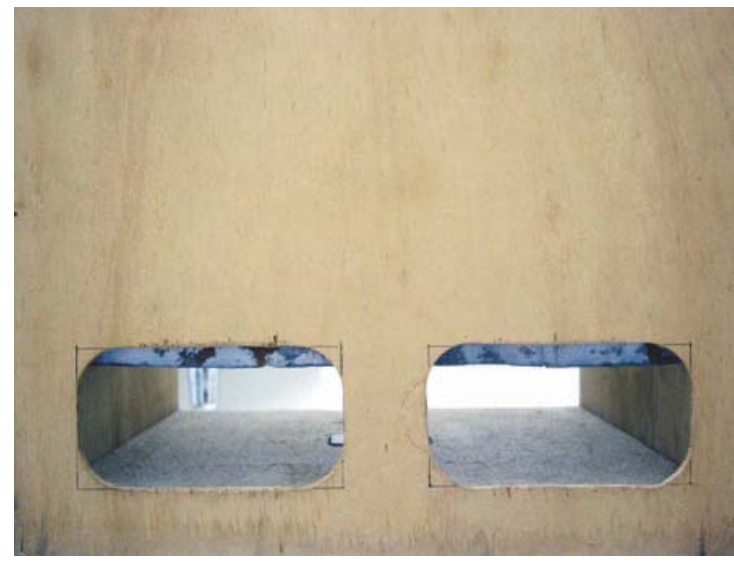

FIGURA 1 - Caixa desenvolvida para tomada radiográfica da região metacarpofalangeana dos polegares. Vista frontal mostrando, em detalhe, a abertura para introdução das mãos.

mado. O estudo foi devidamente aprovado pelo Comitê de Ética da Instituição.

A técnica radiográfica seguiu as orientações propostas por Silva Filho et al. ${ }^{14}$, expondo-se a região da articulação metacarpofalangeana dos polegares esquerdo e direito, por 0,5 segundos, a uma distancia foco-filme de $40 \mathrm{~cm}$.

Os filmes foram processados por um mesmo técnico, utilizando processadora automática com soluções reveladora e fixadora Kodak para raios- $\mathrm{X}$ dentário (Fig. 5).

\section{RESULTADOS E DISCUSSÃO}

Os resultados do presente estudo demonstraram que o osso sesamóide da articulação metacarpofalangeana esteve presente em todos os indivíduos da amostra, totalizando, desta forma, $0 \%$ de agenesia.

Vários métodos para estimação da idade óssea têm sido empregados na prática clínica e em pesquisas científicas ${ }^{1-10,12,13,14}$. Mais recentemente, a telerradiografia lateral também tem sido explorada para a verificação da idade esquelética ${ }^{1,13}$. Entretanto, uma cuidadosa calibração prévia é requerida para confiabilidade do método ${ }^{13}$. O método proposto por Silva Filho et al. ${ }^{14}$ tem se mostrado
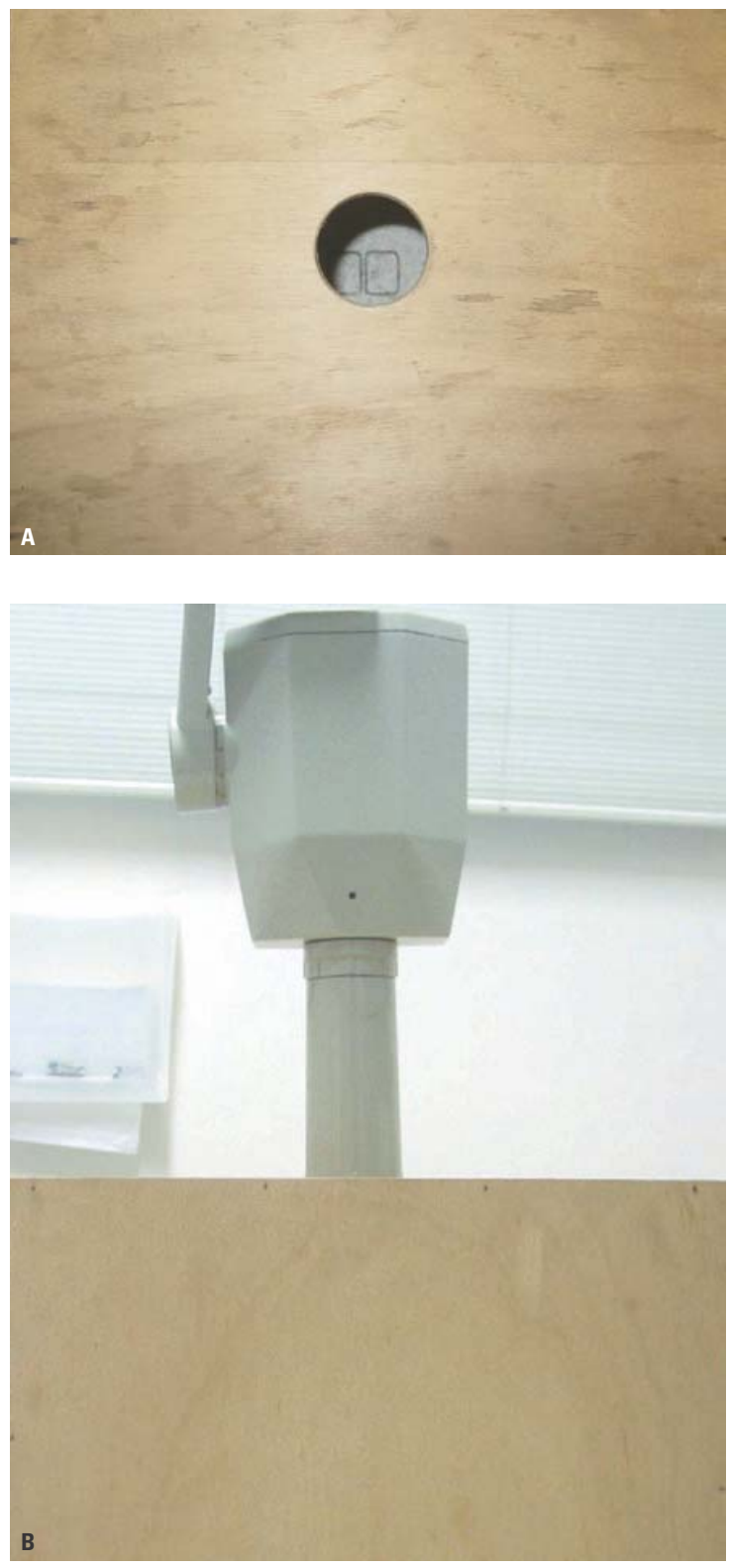

FIGURA 2 - A) Parte superior: orifício para encaixe do aparelho de raios-X. B) Aparelho em posição.

simples e de grande concordância entre examinadores, compatível com o exame carpal classicamente utilizado ${ }^{13}$. O presente trabalho reforça o uso da avaliação do sesamóide adutor, na composição da simplificação do método, como um ótimo parâmetro para pesquisas e também conduta clínicas, corroborando o trabalho de Pileski et al. ${ }^{11}$ 


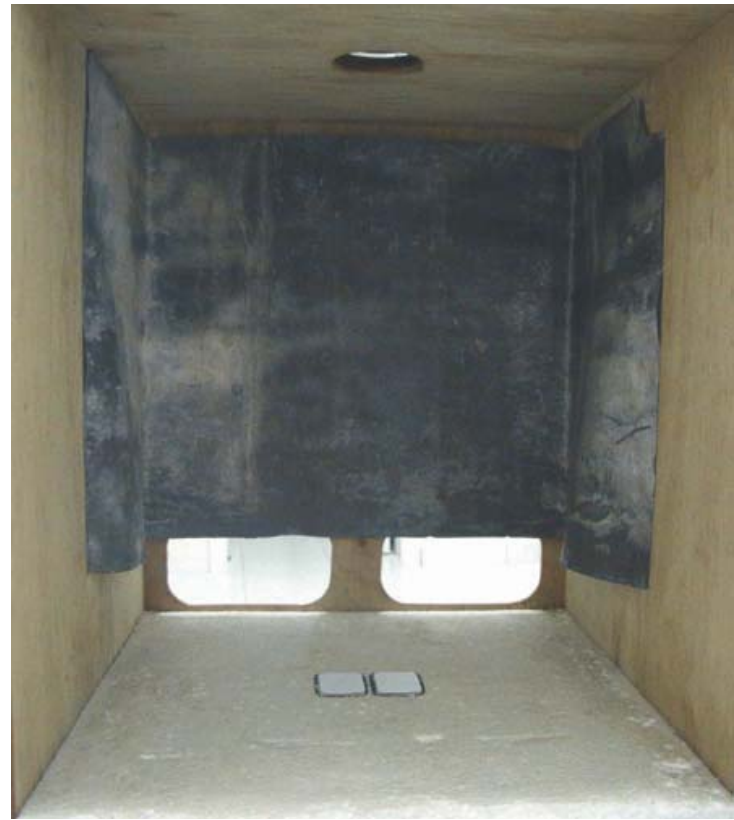

FIGURA 3 - Parte interna revestida com lâmina de chumbo nas faces laterais e frontal.

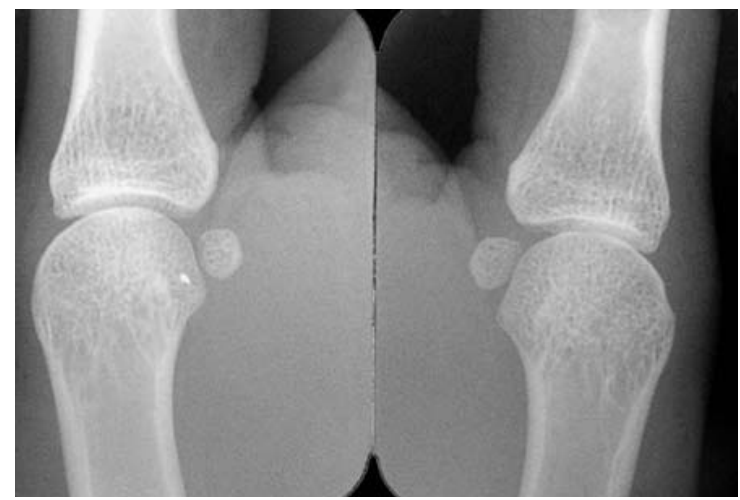

FIGURA 5 - Radiografia periapical do polegar direito e esquerdo de um indivíduo da amostra, evidenciando a presença dos ossos sesamóides.
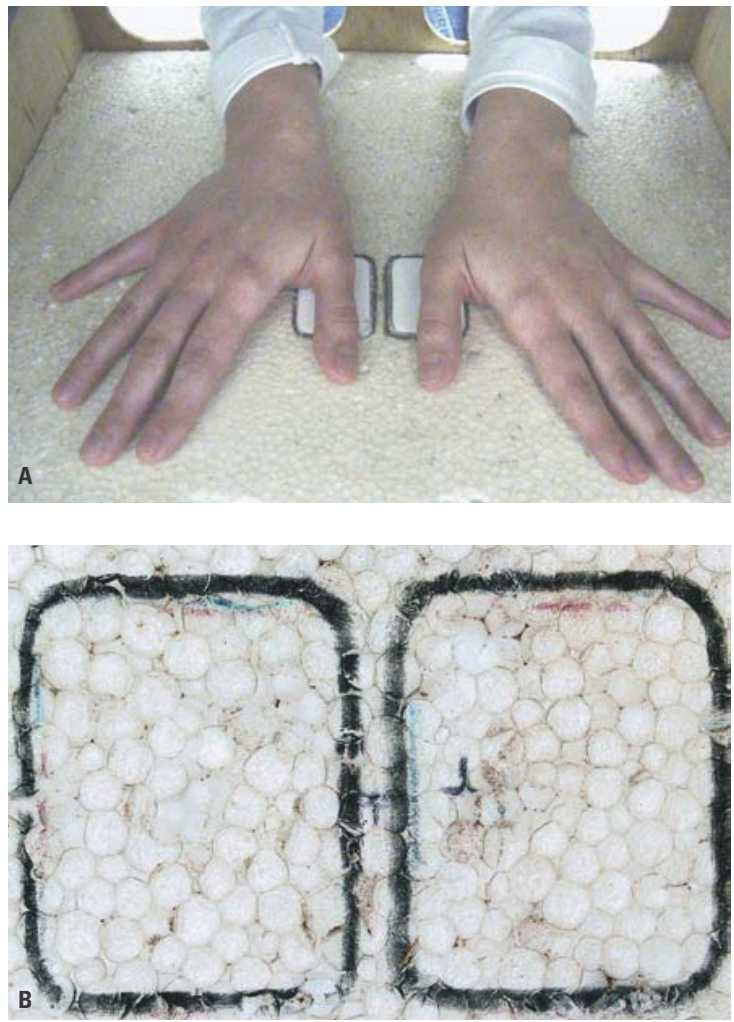

FIGURA 4 - A) Parte interna. B) Detalhe do isopor com marcação para posicionamento correto dos filmes radiográficos.

\section{CONCLUSÃO}

Numa amostra de 500 leucodermas e xantodermas, adultos brasileiros, de ambos os gêneros, não houve agenesia do ossículo sesamóide adutor ( $100 \%$ presente).

\section{AGRADECIMENTOS}

Aos alunos da Faculdade de Odontologia de Lins pela participação na amostra desta pesquisa. 


\title{
Agenesis prevalence of the adductor sesamoid of the thumb in Brazilians adults
}

\begin{abstract}
Aim: the purpose of this study was to verify the percentage of agenesis of sesamoid bone in a Brazilian adult population. Methods: the sample consisted of 329 females and 171 males, with mean age of 22.5 (range from 18 to 33 years). The presence or agenesis of sesamoid bone was evaluated through periapical radiographs taken from the metacarpophalangeal joint of the thumb (left and right) of each subject. Results and conclusion: The results evidenced that the sesamoid bone was present in $100 \%$ of the sample, showing that was not found agenesis in these population.
\end{abstract}

Key words: Maturity indicator. Skeletal development. Sesamoid bone. Carpal index.

\section{REFERÊNCIAS}

1. BACETTI, T.; FRANCHI, L.; MCNAMARA JR., J. An Improved version of the cervical vertebral maturation (CVM). Method for the assessment of mandibular growth. Angle Orthod., Appleton, v. 72, no. 4, p. 316-323, 2002.

2. BJORK, A.; HELM, S. Prediction of the age of maximum puberal growth in body height. Angle Orthod., Appleton, v. 37, p. $134-143,1967$

3. BOWDEN, B. D. Sesamoid bone appearance as an indicator of adolescence. Aust. Orthod., Brisbane, v. 23, p. 242-248, 1971

4. CHAPMAN, S. M. Ossification of the adductor sesamoid and the adolescent growth spurt. Angle Orthod., Appleton, v. 42, p. 236-244, 1972

5. FISHMAN, L. S. Radiographic evaluation of skeletal maturation. A clinically oriented method based on hand-wrist films. Angle Orthod., Appleton, v. 52, p. 88-117, 1982.

6. GRAVE, K. C.:BROWN, T. Skeletal ossification and the adolescent growth spurt. Am. J. Orthod., St. Louis, v. 69, p. 611-619, 1976

7. GREULICH, W. N.; PYLE, S. I. Radiographic atlas of skeletal development of the hand and wrist. 2nd ed. Califórnia: Stanford, 1959

8. HAGG, U.; PANCHERZ, H. Dentofacial orthopaedics in relation to chronological age, growth period and skeletal development: an analysis of 72 male patient with Class II division 1 malocclusion treated with the Herbst appliance. Eur. J. Orthod., Oxford, v. 10, p. 169-176, 1988.
9. HAGG, U.; TARANGER, J. Maturation indicators and the puberal growth spurt. Am. J. Orthod., St. Louis, v. 82, p. 299-309, 1982

10. LEWIS, A. B. et al. Growth of the mandible during pubescence. Angle Orthod., Appleton, v. 52, p. 325-342, 1982.

11. PILESKI, P. C. et al. The relationship of the ulnar sesamoid bone and maximum mandibular growth velocity. Angle Orthod., Appleton, v. 43, p. 162-170, 1973.

12. SAMPAIO, L. L. Avaliação de um método simplificado para estimar a maturação esquelética. 1991. Trabalho de Conclusão de Curso. (Residência Odontológica em Odontopediatria)-Hospital de Pesquisa e Reabilitação de Lesões Lábio-Palatais - USP, Bauru, 1991.

13. SANTOS, S. C. B. N. et al. Avaliação de um método de determinação do estágio de maturação esquelética utilizando as vértebras cervicais presentes nas telerradiografias em norma lateral. Rev. Dental Press Ortodon. Ortop. Facial, Maringá, v. 3, n. 3, p. 67-77, maio/jun. 1998.

14. SILVA FILHO, O. G. et al. Proposta de um método simplificado para avaliação da maturação esquelética. Ortodontia, São Paulo, v. 22, n. 3, p. 33-43, 1989.
Endereço para correspondência

Renata Rodrigues de Almeida-Pedrin

Rua Saint Martin, 35-28

CEP: 17.012-433 - Bauru/SP

E-mail: renatinhaalmeida@uol.com.br 